\title{
RECAUDACIÓN DE IMPUESTOS MUNICIPALES Y SU RELACIÓN CON EL DESARROLLO SOSTENIBLE EN EL DISTRITO DE YARINACOCHA, 2019
}

\author{
MUNICIPAL TAX COLLECTION AND ITS RELATION TO \\ SUSTAINABLE DEVELOPMENT IN THE DISTRICT OF \\ YARINACOCHA, 2019
}

Cheryl Ruiz Bartra ${ }^{1}$

\section{RESUMEN}

La investigación que se da a conocer muestra como propósito determinar en qué medida la recaudación de impuestos se relaciona con el desarrollo sostenible en el distrito de Yarinacocha, 2019; por lo cual, se empleó como metodología de estudio, la investigación no experimental, transversal, correlación causal, con un enfoque cuantitativo. La población estuvo compuesta por 345 trabajadores de la Municipalidad de Yarinacocha, así como la población del distrito. La muestra lo conformó el tipo heterogéneo, para la primera población se empleó el muestreo aleatorio simple y muestreo por conveniencia para la población de Yarinacocha dando un total en ambas muestras de 119 sujetos. Se aplicó dos cuestionarios de acuerdo a la información que se requiere conocer. Se realizó la prueba no paramétrica de Kolmogorov Smirnov, dando como resultado valores Sig. P < 0.05 en la prueba de ajuste, por lo cual, se usó la Estadística inferencial: Para la prueba de las hipótesis, general y específica, se usó la distribución Tau - b de Kendal y la prueba de Rho de Spearman debido a que los valores tuvieron una distribución no normal que facilitó para hacer el análisis. Determinando mediante la prueba del coeficiente Tau b de Kendall un valor de $0,210 * *$ con una significancia de 0,001 , siendo menor al $1 \%$ de significancia estándar $(\mathrm{P}<0.01)$, asimismo se empleó la prueba del coeficiente de Rho de Spearman, indicando un valor de: 0,300**, (**) dando entender en ambos resulta-

1 Correo Electrónico: cherylruizb@hotmail.com Afiliación Institucional: Universidad Cesar Vallejo. 
dos una mayor asociación entre ambas variables, existiendo una correlación positiva baja. Es por ello que se rechaza la hipótesis nula y se acepta la hipótesis general de estudio: Si existe una relación significativa entre la Recaudación de impuestos municipales en el desarrollo sostenible del distrito de Yarinacocha, 2019.

PALABRAS CLAVE: Recaudación de impuestos municipales, desarrollo sostenible.

\begin{abstract}
The research that is released shows the purpose of determining the extent to which tax collection is related to sustainable development in the Yarinacocha district, 2019; Therefore, non-experimental, cross-sectional, causal correlation, with a quantitative approach was used as a study methodology. The population was composed of 345 workers of the Municipality of Yarinacocha, as well as the population of the district. The sample was made up of the heterogeneous type, for the first population simple random sampling and convenience sampling were used for the Yarinacocha population giving a total of both samples for 119. Two questionnaires were applied according to the information that is required to know. The nonparametric Kolmogorov Smirnov test was performed, resulting in Sig values $\mathrm{P}<0.05$ in the fit test, for which the inferential Statistics was used: For the test of the general and specific hypotheses the Tau distribution was used Kendal's b and the Spearman's Rho test because the values had a non-normal distribution that facilitated the analysis. Determining by means of the Kendall Tau_b coefficient test a value of $0.210 * *$ with a significance of 0.001 , being less than $1 \%$ of standard significance $(\mathrm{P}<0.01)$, the Spearman Rho coefficient test was also used, indicating a value of: $0.300 * *,(* *)$ in both results, understanding a greater association between both variables, with a low positive correlation. That is why the null hypothesis is rejected and the general study hypothesis is accepted: If there is a significant relationship between the collection of municipal taxes in the sustainable development of the Yarinacocha district, 2019.
\end{abstract}

KEY WORDS: Collection of municipal taxes, sustainable development.

\title{
INTRODUCCIÓN
}

Se conoce como disposición tributaria la recaudación de impuestos que realiza la entidad estatal a la población de una determinada jurisdicción, debido que el gobierno tiene la obligación de financiar los gastos que contribuyen a reducir las necesidades de la ciudadanía con el fin de lograr un desarrollo social en cada ciudad. De acuerdo a las tasas de morosidad de los tributos municipales se puede deducir que la población no tiene cultura tributaria priorizando todos sus gastos y dejando al final o ignorando el pago de los tributos en su presupuesto mensual. (Rajoy, 2018) 
La recaudación de impuesto municipal contribuye en el desarrollo sostenible de la Amazonía debido a que el aprovechamiento de los recursos disponibles va de la mano con la conservación de la biodiversidad, integrándose en el factor social, reconociendo y valorando la cultura y conocimientos de las comunidades que allí habitan, sostuvo Yolanda Guzmán, del Instituto Peruano de la Amazonía (Andina, 2018) La Municipalidad Distrital de Yarinacocha, es una institución sostenible respetuosa del ambiente, que representa a todos los vecinos a quienes les brinda servicios públicos con eficiencia y eficacia, y su accionar se caracteriza por el trabajo en equipo y la apertura al diálogo, cuenta con una estructura orgánica flexible y recursos humanos idóneos, con honestidad y solvencia moral, infraestructura implementada y equipada con tecnología de punta, que optimizan los sistemas de recaudación tributaria, mejorando las condiciones de vida de la población de Yarinacocha. Según INEI (2017) en el Distrito de Yarinacocha hay más de 97628 habitantes y más de 20026 viviendas, recolectando en un día entre 50 a 100 toneladas de basura, los cuales el 85 por ciento de basura se va al botadero abierto y el 15 por ciento se recicla.

Uno de los objetivos de la Municipalidad de Yarinacocha es representar y velar por las necesidades a todos los vecinos, pero, debido a la falta de indicadores, programas y proyectos no puede promover una adecuada prestación de los servicios públicos locales; generando un avance lento en el desarrollo integral, sostenible y armónico con la participación proactiva y propósito de los centros poblados y grupos étnicos de la Jurisdicción del Distrito de Yarinacocha; afirmando que más de 800 personas hablan quechua, más de 73 hablan Aymará, más de 128 son Asháninka, 5052 personas aproximadamente hablan otras lenguas maternas, 73068 hablan castellano y 82 personas hablan otros idiomas extranjeros y 86 personas son sordomudos. (INEI, 2017)

La recaudación de los impuestos es necesario, a mayor recaudación se puede obtener los recursos para invertir en: educación, salud, seguridad, justicia, obras públicas, combate a la pobreza y el impulso a los sectores económicos que son fundamentales para el crecimiento de la economía en el Distrito de Yarinacocha; debido a la importancia de los impuestos por la Municipalidad de Yarinacocha se manifiesta que hasta el 30 de septiembre con respecto al Impuesto Predial se recaudó 2970237.29 , con respecto al impuesto de Alcabala se recaudó 555 091.51, asimismo el impuesto a los espectáculos públicos no deportivos se recaudó 320898.63 y por el pago de derechos y tasas administrativas se recaudó 690 451.29. Existe un crecimiento en la recaudación entre el año 2018 y 2019, se 
puede afirmar que lo recaudado infiere en la recaudación de basura, limpieza de calles, seguridad ciudadana, etc.

Existen múltiples estudios donde se analizan las variables "Recaudación de impuestos municipales" y "desarrollo sostenible", desde un ámbito local, nacional e internacional, con fin de analizar y conocer su despliegue en los diferentes entornos de estudio, por lo cual, se investigó los repositorios de las diversas universidad y se extrajo investigaciones científicas que coincidieron con las variables de estudio, según Chingay (2019), el tipo de metodología que se emplea en esta investigación es de tipo descriptiva enfocándose en la recaudación de impuestos de la Municipalidad Distrital de Huarmaca, buscando explicar el movimiento de los recursos municipales en relación a los pagos que hacen los pobladores de dicho distrito, conociendo cuan comprometidos están en los pagos, por otro lado reconocer las características, y las facilidades de pago que brinda la municipalidad a sus pobladores, extrayendo información de todo lo que tenga que ver con la recaudación de impuestos o tributaria, este trabajo de investigación tiene un diseño no experimental, sino la de recoger información de las áreas de recaudación de impuestos aplicando un cuestionario para tener un diagnóstico institucional. Se llegó a concluir que el municipio de Huarmaca no tiene una fluctuación adecuada en la recaudación de impuestos, situación que puede deberse a una inadecuada gestión al no tomar en cuenta estrategias que puedan concientizar y dar información a la población en el pago de los tributos y los beneficios que esto le trae para la población.

Dentro de este marco, Arrioja, (2003) en Teoría de la Relación de Sujeción hace mención a la particularidad que tiene el pago de un tributo, la cual es un aspecto óseo, que se sostiene al poder de un sistema de gobierno. Dicho de otro modo, el tributo que le corresponde a una persona pagar no está sujeto a la necesidad de recibir un beneficio a cambio, sino por una sencilla orden de poder y por parte de cada ciudadano como una simple acción de sumisión y de obediencia a los poderes del Estado, los cuales están encargados del bienestar de la ciudadanía. En oposición a ello, se pasó a formular distintas convergencias y críticas como el razonamiento, no solo por parte de los pobladores que se vuelven súbditos de los tributos, sino también de las personas que provienen de otros países.

El Sistema Tributario Municipal del Perú abarca todo un conglomerado de impuestos, tasas y contribuciones, dispuestos en el Texto Único Ordenado de la Ley de Tributación Municipal. Para continuar se describirá todos y cada uno de ellos. 
Impuesto Predial: El ámbito de este impuesto se da a nivel distrital, la periodicidad es anual y grava el valor que tienen los predios ya sean estos urbanos o rústicos.

Impuesto de Alcabala: Este impuesto es de actuación inmediata y grava todos aquellos traspasos de propiedad de inmuebles urbanos o rústicos, ya sean estos a título oneroso o gratuito, o cualquier otra forma. Si una Municipalidad posee un fondo de inversión el alcance de este impuesto se puede dar a nivel provincial, y de no ser el caso, su alcance sería a nivel distrital.

Impuesto al Patrimonio Vehicular: El ámbito de este impuesto es provincial, la periodicidad con la que se tributa es anual y grava los vehículos, automóviles, camionetas, station wagons, camiones, buses y ómnibus que sean de propiedad de las personas y que tengan una antigüedad no mayor de tres (3) años de haberse inscrito en el Registro de Propiedad Vehicular.

Impuesto a las Apuestas: Este impuesto presenta un alcance provincial, la periodicidad con la que se tributa es mes a mes y grava todas las entradas que reciban aquellas empresas que organizan eventos hípicos y semejantes en donde se realicen apuestas.

Impuesto a los Juegos: El periodo para tributar este impuesto es de manera mensual y grava las actividades realizadas y que tengan relación con los juegos, tales como loterías, bingos y rifas, así como las ganancias obtenidas en los juegos de azar. Respecto a los juegos de bingo, rifas, sorteos y semejantes, así también como para el juego de pinball, juegos de video y otros juegos electrónicos, tienen un alcance a nivel distrital; y en caso de loterías y otros tipos de juegos de azar, su ámbito es a nivel provincial.

Impuesto a los Espectáculos Públicos no Deportivos: El ámbito de este impuesto se da a nivel distrital y grava aquellos abonos por presenciar y se participante en los espectáculos públicos no deportivos ya sean en locales y/o parques cerrados. Desde inicios del año 2008, la tasa que se debe aplicar a la base imponible del impuesto para conciertos musicales en general, espectáculos de folclor nacional, teatro cultural, zarzuela, conciertos de música clásica, ópera, opereta, ballet y circo es del 0 por ciento.

De manera sustancial, se dice que el Desarrollo Sostenible es aquel curso de cambio mediante el cual la explotación de los recursos, la administración correcta de las inversiones, la guía del desarrollo tecnológico y la permuta institucional 
deben armonizar y reforzar su potencial presente y futuro, con la determinación de encargarse de aquellas necesidades y aspiraciones que tienen las personas (Carvalho, 1993). "Del mismo modo para que exista un desarrollo sostenible requiere:

$\checkmark$ Que la totalidad de personas hayan cubierto aquellas necesidades básicas y les sean facilitadas las oportunidades para lograr el cumplimiento de sus aspiraciones a una mejor calidad de vida.

$\checkmark$ El fomento de valores que conserven los patrones de consumo adentro de los parámetros que las posibilidades económicas ofrecen y que todos puedan anhelar a ellos de forma racional.

$\checkmark$ Que un aumento económico en las regiones donde sus necesidades no son consideradas. Donde sí lo son, el desarrollo sostenible es similar con el aumento económico, ya que dicho aumento plasma los vastos principios sobre la sostenibilidad y la no explotación de los otros.

$\checkmark$ Que el registro por la devastación de recursos no renovables custodie todas las alternativas futuras posibles al máximo.

$\checkmark$ El cuidado de las variedades de animales y vegetales.

$\checkmark$ Disminuir las impresiones desfavorables de la calidad del aire, del agua y de distintos elementos naturales, con la finalidad de proteger la integridad global del ecosistema.

$\checkmark$ Que se reanuden las políticas internacionales establecidas por los países industrializados, con el fin de buscar el crecimiento, el comercio y la inversión." (Carvalho, 1993)

Dada las circunstancias establecidas en los párrafos anteriores, estructurado por la necesidad, importancia, justificación, problemas antecedentes y teoría, se plantea la siguiente pregunta ¿En qué medida los impuestos municipales se relacionan con el desarrollo sostenible del distrito de Yarinacocha, 2019?, por lo cual, la investigación se justifica mediante un argumento teórico, debido a que la finalidad es contribuir al discernimiento existente sobre las variables en estudio: "Recaudación de impuesto municipal" y "Desarrollo Sostenible", asimismo con sus herramientas de calificación de la obtención de competencias de supervisión y análisis científicas en la población de Yarinacocha, siendo los resultados direccionados a una propuesta para ser integrados como conocimientos en la gestión pública, debido que se comprobaría que las variables en estudio contribuyen en el desarrollo sostenible de la ciudadanía de Yarinacocha. 
Con respecto a la justificación práctica se puede afirmar que el estudio se ejecuta con la necesidad de cambiar la priorización de programas y/o proyectos para lograr un desarrollo sostenible donde los más beneficiados resultan ser los habitantes del distrito de Yarinacocha. Así también se justifica metodológicamente, debido que se elaboró y se aplicó para cada variable medida e indicadores de acuerdo a una línea de estudio que fue corroborado a través de la validez y la confiabilidad, de modo tal que los resultados resulten verídicos y puedan contribuir con próximas investigaciones en las variables mencionadas.

El propósito de la investigación es muy importante en el estudio, debido que integra una guía para llevar a cabo el estudio ya sea en un corto, mediano o largo plazo, permitiendo un orden en el desarrollo de los resultados, por lo cual, como objetivo general se busca determinar en qué medida la recaudación de impuestos se relaciona con el desarrollo sostenible del distrito de Yarinacocha, 2019; así también se señala que la hipótesis general es conocer si existe una relación significativa entre la Recaudación de impuestos.

\section{MÉTODOS}

Se optó por el modo no experimental, por su finalidad es aplicada y/o empírica, por su profundidad o carácter es correlacional causal, por su naturaleza es cualitativa con un enfoque cuantitativo, por el alcance es transversal.

La población 1 se encuentra constituida por 345 trabajadores de la Municipalidad de Yarinacocha. La población 2, lo conforman todos los contribuyentes del distrito de Yarinacocha. Para ambos casos, la muestra es de 119 personas.

Se compiló la información numérica a través de documentos impresos, así como contenido de los archivos virtuales de las oficinas de recaudación de la Municipalidad de Yarinacocha, mediante el contenido recopilado de las fuentes de extracción de datos, se otorgó alternativas prácticas de solución ante los problemas descritos en la introducción a través de múltiples técnicas y métodos estadísticos para comprobar la hipótesis de disertación. (Hernández et al, 2015)

Para el análisis de los resultados se organizaron en:

Estadística descriptiva. Estadísticos descriptivos: Se empleó la desviación estándar, media aritmética varianza que se logró interpretar mediante tablas y figuras.

Inferencia estadística. A través del instructivo estadístico de Morales 
(2015) se realizó la prueba no paramétrica de Kolmogorov Smirnov, dando como resultado valores Sig. P $<0.05$ en la prueba de ajuste, por lo cual, se usó la Estadística inferencial: Para la prueba de las hipótesis general y específica se usó la distribución Tau - b de Kendal y la prueba de Rho de Spearman debido a que los valores tuvieron una distribución no normal que facilitó para hacer el análisis.

Para el análisis de los resultados se organizaron en:

Estadística descriptiva. Estadísticos descriptivos: Se empleó la desviación estándar, media aritmética varianza. Logrando interpretar mediante tablas y figuras estas estadísticas.

Inferencia estadística. A través del instructivo estadístico de Morales (2015).

\section{RESULTADOS}

A través del presente Capítulo, se plasma la recopilación de los datos extraídos por la muestra en investigación, siguiendo el orden sistemático de los objetivos con el fin de aceptar o rechazar las hipótesis de estudio, es por ello, que se empleó pruebas no paramétricas, así como: la prueba del coeficiente de Tau-b de Kendall, con el propósito de conocer el vínculo entre variables y dimensiones.

Según la opinión de la muestra del talento humano de la Municipalidad de Yarinaocha, se observa que la variable 1 Recaudación de impuesto municipales predomina en un nivel nada importante con una frecuencia de 119 (100 por ciento), asimismo se visualiza a la variable 2 desarrollo sostenible, la cual tiene mayor incidencia en un nivel moderadamente importante con una frecuencia de 109 (91,6 por ciento)

La variable 1 recaudación de impuestos municipales, tiene cincos componentes importantes para su desarrollo y de acuerdo a la muestra de 119 , señalaron que se encuentran en un nivel moderadamente importante, predominando la dimensión "cultura tributaria" con una frecuencia de 89 ( 74,8 por ciento), seguido de la dimensión "beneficios tributario" con una frecuencia de 74 (62,2 por ciento), en tercer lugar figura la dimensión "eficacia del sistema" con una frecuencia de 64 (53,8 por ciento), seguido de la dimensión "acceso a servicio de la administración tributaria" con una frecuencia de 53 (44,5 por ciento) y por último la dimensión "grado de información al contribuyente" con una frecuencia de 45 (37,8 por ciento). 
Conforme a los resultados de la percepción del talento humano de la Municipalidad de Yarinacocha, de las tres dimensiones de la variable desarrollo sostenible figura en primer lugar la dimensión "aspecto económico" con una frecuencia de 78 ( 65,5 por ciento) en un nivel de moderadamente importante, seguido de la dimensión "aspecto ecológico" con una frecuencia de 73 (61,3 por ciento) en un nivel de importante, en tercer lugar la dimensión "aspecto ecológico" con una frecuencia de 73 (61,3 por ciento) en un nivel importante.

\section{DISCUSIÓN}

De acuerdo a las tasas de morosidad de los tributos municipales se puede deducir que la población no tiene cultura tributaria priorizando todos sus gastos y dejando al final o ignorando el pago de los tributos en su presupuesto mensual (Rajoy, 2018). La recaudación de impuesto municipal contribuye en el desarrollo sostenible de la Amazonía debido que el aprovechamiento de los recursos disponibles va de la mano con la conservación de la biodiversidad, integrándose en el factor social, reconociendo y valorando la cultura y conocimientos de las comunidades que allí habitan, sostuvo Yolanda Guzmán, del Instituto Peruano de la Amazonía (Andina, 2018)

La Municipalidad de Yarinacocha dentro de sus objetivos de gestión tiene la finalidad de emplear un desarrollo sostenible el cual es la respuesta a los problemas sociales y ambientales de la jurisdicción, debido que el desarrollo satisfará las necesidades actuales de las personas, sin comprometer la capacidad de las próximas generaciones para satisfacer las suyas. La responsable edil considera que el desarrollo genera bienestar para todas las personas, por lo cual, es una tarea multisectorial donde, sin duda, su gestión tiene un rol protagónico puesto que desde la gestión pública corresponde entender cuáles son los paradigmas de un tipo de desarrollo más inclusivo y con menos impacto ambiental, para diseñar políticas públicas acordes con las diversas necesidades de la población.

Según la opinión de la muestra del talento humano de la Municipalidad de Yarinacocha, se observa que la variable 1 Recaudación de impuesto municipales predomina en un nivel nada importante con una frecuencia de 119 (100 por ciento), asimismo se visualiza a la variable 2 desarrollo sostenible, la cual tiene mayor incidencia en un nivel moderadamente importante con una frecuencia de 109 (91,6 por ciento), según la tabla 7, se visualiza la percepción de las variables Recaudación de impuestos municipales y Desarrollo sostenible por la muestra de 
119 trabajadores de la Municipalidad de Yarinacocha, los cuales fueron procesados en el SPSS utilizando las pruebas del coeficiente Tau_b de Kendall cuyo valor es de $0,210^{* *}$ con una significancia de 0,001 , que es menor al $1 \%$ de significancia estándar $(\mathrm{P}<0.01)$, asimismo se empleó la prueba del coeficiente de Rho de Spearman, indicando un valor de: $0,300 * *,(*)$ dando entender en ambos resultados hay una mayor asociación entre ambas variables, existiendo una correlación positiva baja (Débil). Es por ello que se rechaza la hipótesis nula y se acepta la hipótesis general de estudio: Si existe una relación significativa entre la Recaudación de impuestos municipales en el desarrollo sostenible del distrito de Yarinacocha, 2019.

También se analiza el estudio de Chávez (2015), donde indica que la participación por parte de la ciudadanía en el gasto de sus impuestos les brinda el conocimiento necesario sobre los beneficios que resultan de las contribuciones que realizan, ayudándolos también a poseer una cultura tributaria, la cual apoya a la mayor parte de la población a agilizar la realización de las metas de la Municipalidad del Cantón Paztaza contribuyendo a la mejora del servicio de atención y las necesidades de los pobladores. Concluyendo de todos los estudios que la recaudación de los tributos municipales en paralelo con un gasto público priorizando necesidades disminuye las brechas establecidas por el gobierno, lo que genera un desarrollo social.

Dentro de este marco, en Teoría de la Relación de Sujeción hace mención a la particularidad que tiene el pago de un tributo, la cual es un aspecto óseo, que se sostiene al poder de un sistema de gobierno. Dicho de otro modo, el tributo que le corresponde a una persona pagar no está sujeto a la necesidad de recibir un beneficio a cambio, sino por una sencilla orden de poder y por parte de cada ciudadano como una simple acción de sumisión y de obediencia a los poderes del Estado, los cuales están encargados del bienestar de la ciudadanía. En oposición a ello, se pasó a formular distintas convergencias y críticas como el razonamiento, no solo por parte de los pobladores que se vuelven súbditos de los tributos, sino también de las personas que provienen de otros países (Arrioja, 2003).

La variable 1 recaudación de impuestos municipales, tiene cincos componentes importantes para su desarrollo y de acuerdo a la muestra de 119 , señalaron que se encuentran en un nivel moderadamente importante, predominando la dimensión "cultura tributaria" con una frecuencia de 89 ( 74,8 por ciento), seguido de la dimensión "beneficios tributario" con una frecuencia de 74 (62,2 por 
ciento), en tercer lugar figura la dimensión "eficacia del sistema" con una frecuencia de 64 (53,8 por ciento), seguido de la dimensión "acceso a servicio de la administración tributaria" con una frecuencia de 53 (44,5 por ciento) y por último la dimensión "grado de información al contribuyente" con una frecuencia de 45 ( 37,8 por ciento).

Asimismo, en el estudio de Huanancuni (2018), tuvo el propósito de establecer la relación del manejo tributario de la administración y el pago de los impuestos, asimismo informar sobre el provecho que traerá a los ciudadanos el pago de sus impuestos, en ese sentido para apresurar la marcha del desempeño tributario en un futuro no muy lejano de la Municipalidad se debería realizar un estudio que dé a entender a los ciudadanos el impacto que trae la gestión municipal con el fin de ayudar a aumentar la efectividad y la fluctuación de los pagos prediales, colaborando no solo con el incremento de los ingresos de la Municipalidad por parte de los contribuyentes, sino también a ofrecer mejores servicios a los ciudadanos. Después del análisis se puede concluir que a nivel nacional hace falta una reforma de política nacional tributaria. El ministrito de Economía y Finanzas, manifiesta que comparando los niveles de tributación municipal de los países que conforman la Alianza del Pacífico, la recaudación nacional figura como una de las más bajas. Los municipios tienen como función la cobranza, gestión y verificación del impuesto, a pesar de la función otorgada no puede transformar los requisitos para determinar la tasa del impuesto y como órganos de gobierno local son llamados a promover el desarrollo en su comunidad y prestar servicios básicos dentro de su jurisdicción. Por ello se hace necesario apostar por una mayor y mejor orientación tributaria municipal a la gente.

\section{CONCLUSIONES}

Primera: Se determina mediante las pruebas del coeficiente Tau_b de Kendall cuyo valor es de $0,210 * *$ con una significancia de 0,001 , siendo menor al $1 \%$ de significancia estándar $(\mathrm{P}<0.01)$, asimismo se empleó la prueba del coeficiente de Rho de Spearman, indicando un valor de: $0,300 * *,(* *)$ dando entender en ambos resultados hay una mayor asociación entre ambas variables, existiendo una correlación positiva baja (Débil). Es por ello que se rechaza la hipótesis nula y se acepta la hipótesis general de estudio: Si existe una relación significativa entre la Recaudación de impuestos municipales en el desarrollo sostenible del distrito de Yarinacocha, 2019. 
Segunda: Se determina a través de los resultados de la prueba del coeficiente Tau_b de Kendall un valor de 0,159* con una significancia de 0,026, que es menor al $5 \%$ de significancia estándar $(\mathrm{P}<0.05),\left(^{*}\right)$ dando entender que existe una asociación entre dimensión y variable; asimismo se empleó la prueba del coeficiente de Rho de Spearman, indicando una puntuación de: 0,195*, con una significancia de 0,034, lo que quiere decir que hay una correlación positiva muy baja (muy débil).

Tercera: Se determina mediante la prueba del coeficiente Tau_b de Kendall cuyo valor es de $0,215 * *$ una significancia de 0,002 , que es menor al $1 \%$ de significancia estándar $(\mathrm{P}<0.01)$, asimismo se empleó la prueba del coeficiente de Rho de Spearman, indicando un valor de: 0,282**, (**) dando entender en ambos resultados hay una mayor asociación entre ambas variables, existiendo una correlación positiva baja (débil).

Cuarta: Se determina a través de la prueba del coeficiente Tau b de Kendall cuyo valor es de $0,199 * *$ con una significancia de 0,003 , que es menor al 1\% de significancia estándar $(\mathrm{P}<0.01)$, asimismo se empleó la prueba del coeficiente de Rho de Spearman, indicando un valor de: 0,261**, (**) dando entender en ambos resultados hay una mayor asociación entre ambas variables, existiendo una correlación positiva baja (débil).

Quinta: Se determina por medio de la prueba del coeficiente Tau_b de Kendall un valor de $0,150 *$ con una significancia de 0,027 , que es menor al 5\% de significancia estándar $(\mathrm{P}<0.05),(*)$ dando entender que existe una asociación entre dimensión y variable; asimismo se empleó la prueba del coeficiente de Rho de Spearman, indicando una puntuación de: 0,201*, con una significancia de 0,029, lo que quiere decir que hay una correlación positiva baja (débil).

Sexta: $\quad$ Se determina por la prueba del coeficiente Tau b de Kendall cuyo valor es de $0,144^{* *}$ con una significancia de 0,036 , que es menor al $5 \%$ de significancia estándar $(\mathrm{P}<0.05)$, asimismo se empleó la prueba del coeficiente de Rho de Spearman, indicando un valor de: 0,199**, (**) dando entender en ambos resultados que hay una mayor asociación entre ambas variables, existiendo una correlación positiva muy baja (muy débil). Es por ello que se rechaza la hipótesis nula y se acep- 
ta la hipótesis especifica de estudio: Si existe una relación significativa entre la eficacia del sistema con el desarrollo sostenible del distrito de Yarinacocha, 2019.

Séptima: Se determina a través de la prueba del coeficiente Tau b de Kendall cuyo valor es de $0,214^{* *}$ con una significancia de 0,001 , que es menor al $1 \%$ de significancia estándar $(\mathrm{P}<0.01)$, asimismo se empleó la prueba del coeficiente de Rho de Spearman, indicando un valor de: 0,290**, $(* *)$ dando entender en ambos resultados hay una mayor asociación entre ambas variables, existiendo una correlación positiva baja (débil). Es por ello que se rechaza la hipótesis nula y se acepta la hipótesis especifica de estudio: Si existe una relación significativa entre la recaudación de impuestos municipales con el aspecto económico del desarrollo sostenible del distrito de Yarinacocha, 2019.

Octava: Se determina según la prueba del coeficiente Tau b de Kendall cuyo valor es de $0,177 * *$ con una significancia de 0,008 , que es menor al $1 \%$ de significancia estándar $(\mathrm{P}<0.01)$, asimismo se empleó la prueba del coeficiente de Rho de Spearman, indicando un valor de: 0,247**, (**) dando entender en ambos resultados hay una mayor asociación entre ambas variables, existiendo una correlación positiva baja (débil). Es por ello que se rechaza la hipótesis nula y se acepta la hipótesis especifica de estudio: Si existe una relación significativa entre la recaudación de impuestos municipales con el aspecto ecológico del desarrollo sostenible del distrito de Yarinacocha, 2019.

\section{REFERENCIAS BIBLIOGRÁFICAS}

Arrioja , A., (2003) "Teoría de la relación de sujeción" Derecho fiscal. Editorial Themis, $\mathrm{N}^{\circ}$ de edición 17. Colección de textos universitarios. México.

Andina, (2018) "Desarrollo sostenible de la Amazonía es viable si se conserva biodiversidad y se integra factor social". 01 de agosto. Andina. Lima - Perú. Recopilado en: https://andina.pe/ agencia/noticia-desarrollo-sostenible-de-amazonia-es-viable-si-se-conservabiodiversidad-y-se-integra-factor-social-187380.aspx

Carvalho H. (1994) “A participaçao e organizaçao consentida como uma das dimensões da cidadania.Informe integral (versión preliminar). Brasilia. IICA/SEPLAN. 1993. Padrões de sustentabilidade: Uma medida para o desenvolvimento sustentável (mimeo). Curitiba. 
Cheryl Ruiz Bartra

Chávez, A., (2015) "La cultura tributaria y su incidencia en la recaudación de impuestos municipales del gobierno autónomo descentralizado del Cantón Pastaza" Para Obtener el Grado de Magister en Tributación y Derecho Empresarial Ambato - Ecuador.

Chingay, (2019) "Recaudación tributaria en el distrito de Huarmaca, 2018"- Para optar el grado de Bachiller en Contabilidad. Universidad Señor de Sipán. Pimentel - Perú.

INEI, (2017) “Ucayali Compendio Estadístico 2017”. Instituto Nacional de Estadifica e Informática. Censo Nacional 2017. Lima - Perú. Recopilado en: http://www.muniyarinacocha.gob.pe/ pdf/INEI/COMPENDIO\%202017.pdf

Oliva, C., (2018) "La recaudación tributaria peruana es una de las más bajas de América Latina". Prensa. 02 de octubre. Prensa. Universidad del Pacífico. Lima-Perú. Recopilado en: https://www.up.edu.pe/prensa/noticias/la-recaudacion-tributaria-peruana-es-una-de-lasmas-bajas-de-america-latina

Rajoy, M., (2018) "España se convierte en el cuarto país de la eurozona que menos recauda". Economía. 24 de marzo. España. Recopilado en: https://www.elconfidencial.com/economia/2018-0424/espana-cuarto-pais-europa-menos-recauda-impuestos_1554089/ 